\title{
Future of colorectal surgery
}

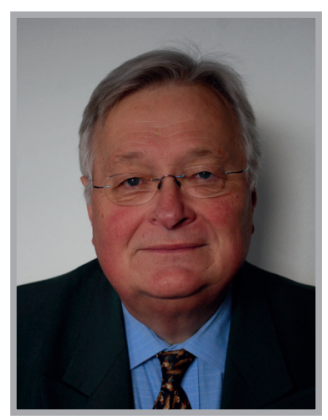

Lars Påhlman*: Lars Påhlman was born in 1946. He graduated at Sigtunaskolan, Sweden, in 1966 and from the Medical School at Uppsala University in 1973, before moving to the District General Hospital in Falun where he had his surgical training. Professor Påhlman moved back to Uppsala in 1979 as a consultant surgeon and finished his PhD thesis on rectal cancer in 1985. In 1987, he had a position as a Reader in Surgery at Uppsala University and since 1999 as a Professor of Surgery at the same university. Professor Påhlman's main interests have been colorectal cancer, function disorders, diverticulosis and diverticulitis. He has been responsible for numerous randomized trials and has had more than 20,000 patients in different randomized trials regarding radiotherapy and rectal cancer, different adjuvant and palliative treatment in colorectal cancer, diverticulosis and diverticulitis, fissures, hemorrhoids and fistulas. His main research is administration of large randomized trials, including translational research. Professor Påhlman speaks to Launch Editor, Charlotte Barker, about colorectal cancer and surgery.

\section{Q What led you to specialize in the area of colorectal cancer early on in your career?}

It is a long history! When I moved from a smaller hospital to Uppsala University Hospital I started to work with rectal cancer in my thesis, more than 30 years ago. From that early interest, I decided to become a colorectal surgeon, dealing with colorectal cancer as well as benign disease. I have published many studies on benign disease but my main research focus is on colorectal cancer.

Q What advice would you give to a young surgeon starting out in the field? I would want them to be colorectal surgeons of course! Obviously, I am a bit biased after 30 years in the field but it is a fascinating specialism and one you can use in most hospitals. We have the opportunity to operate a lot, it is daytime surgery with few emergency cases and because we are dealing with such a common disease you have plenty of patients to study, so enrollment for clinical trials is relatively easy. Upper GI and biliary tract surgery is now concentrated to a few major centers in most countries but colorectal cancer is such a common disease that you can work on it anywhere.

I was raised in a system where surgeons specialize in specific organs, rather than having surgical oncologists, like in Holland or the UK. Both systems have their proponents but I find it hard to understand how you can operate on multiple organs as a surgeon, because as

*University Hospital, Uppsala, Radiology \& Clinical Immunology, Section of Radiology, Uppsala, SE-75105, sweden; lars.pahlman@surgsci.uu.se

\section{News \& Views}

News

Journal Watch

Ask the Experts

Interview

\footnotetext{
"In my ideal world, we should have around 50 units worldwide in a consortium who are committed to join whatever trial we decide on."
}

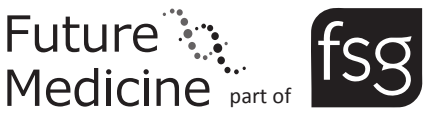




\section{"I believe that we have \\ to focus more on quality control and quality of care. We focused on quality in Norway and \\ Sweden and have changed the outcome of rectal cancer dramatically."}

a colorectal surgeon you learn so much from operating on benign disease that you can then apply to cancer. I have learnt a lot about how the bowel works while treating benign disease, and this has changed my work in colorectal cancer.

Q What has been your greatest achievement to date in your work?

The biggest achievement is that I have implemented preoperative radiotherapy in rectal cancer. I was responsible for two large trials in Sweden, looking at pre- versus post-operative radiation and the now famous Swedish Rectal Cancer Trial, showing the effectiveness of preoperative radiotherapy compared with surgery alone.

Even now, the Swedish schedule of $5 \times 5$ Gy is not used everywhere (it is not financially beneficial because you treat the patient just five-times and not 25-times so some clinicians have been reluctant to switch), but I think it is coming closer.

\section{Q You have been involved in numerous} large-scale clinical trials: what are the most important things to consider when launching a trial of this type?

The most important thing is to have a thrilling idea. Then you have to test that idea on colleagues - if it becomes clear that lots of people agree that it is a good idea then you should write a very preliminary protocol. It usually takes around 1.5 years before everyone is satisfied with the protocol and then you can start looking for centers to join the trial.

We have just finished a protocol and are now starting a huge trial in advanced rectal cancer patients, looking at preoperative chemotherapy, with a Dutch group and Swedish group. I presented the protocol at a meeting in Copenhagen a month ago and already there are several centers who want to join the trial.

However, it is still a problem to get everyone on board with trials. Too many patients are not studied. In Sweden, we have 1500 rectal cancer patients per year, so we should have new trials starting every 6 months. In my ideal world, we should have around 50 units worldwide in a consortium who are committed to join whatever trial we decide on. That would mean we could run the trial as soon as the protocol is agreed and once patients have been recruited to one trial, we would have the protocol lined up to start recruiting for the next trial. The ideal would be for most patients to go into some sort of trial.

Most doctors who do not join us in trials claim that they have no research nurses or lack the funds to run a trial. Of course, these are valid reasons but I think it is wrong to always let others run all the trials and simply wait for the results - why not join us to add patients to the trial?

Q What do you think will be the next major advance in colorectal cancer management? There have been so many crystal ball predictions over the years! There are so many ideas that we do not have enough patients to test them.

Personally, I believe that we have to focus more on quality control and quality of care. We focused on quality in Norway and Sweden and have changed the outcome of rectal cancer dramatically. Since Norway started the same rectal cancer program as we have in Sweden, they have saved 2500 Norwegians lives. This has been achieved by using quality control to ensure good surgical and nonsurgical treatment, at a cost of around $€ 750$ per life saved, compared with a cost of $€ 11,000$ per life saved with adjuvant chemotherapy. This is something that should be implemented worldwide. There is too much focus on new drugs: new drugs are important but we must maximize the effect of the treatments we already have as well.

Screening is also important, but the problem is that most of the patients we are screening are the people who eat healthily, go jogging, attend their screening appointments and do everything as they should. But those people who have risk factors for colorectal cancer, who drink and smoke for example, who we really want to screen, never come to the screening programs: a problem for all cancers. Getting these people into screening programs would be a major advance.

Q The Swedish healthcare system is often held up as an example for high standards of care: why do you think that is?

We have a completely transparent quality registry in Sweden. I think that is the way to go. It is a huge debate in other countries - for example, after the UK published results from heart surgery and several surgeons had to stop operating, it is now difficult to find surgeons who want to take difficult cases. In Sweden, we 
are living with a transparent quality registry, scoring all sorts of different outcome factors, for example, time to chemotherapy, local recurrence rate and survival. If the center does not reach the expected level and does not improve, it is ultimately shut down.

Q What will be the focus for your work over the next few years?

Within a year or two, I plan to retire but for now I am heavily involved in running the major trials I mentioned earlier. I plan to continue to be involved in the trials even after I retire from clinical work, because I think that those large-scale trials are key to improving patient outcomes.

\section{Financial \& competing interests disclosure}

$L$ Pahlman has no relevant affliations or financial involvement with any organization or entity with a financial interest in or financial conflict with the subject matter or materials discussed in the manuscript. This includes employment, consultancies, honoraria, stock ownership or options, expert testimony, grants or patents received or pending, or royalties.

No writing assistance was utilized in the production of this manuscript. 